\title{
Quiste endodérmico supratentorial: caso clínico y revisión de la literatura
}

\author{
J.M. Garbizu; O. Mateo-Sierra; B. Iza; F. Ruiz-Juretschke y J.M. Pérez-Calvo
}

Servicio de Neurocirugía. Hospital General Universitario Gregorio Marañón. Madrid.

\section{Resumen}

Los quistes endodérmicos que afectan al sistema nervioso central son lesiones expansivas muy poco frecuentes que se sitúan con mayor frecuencia a nivel espinal. Existen poco casos de localización intracraneal descritos en la literatura, la mayoría de ellos en la fosa posterior. Su etiopatogenia permanece aún desconocida.

Presentamos el caso de un varón de 62 años que debuta con un cuadro de desorientación y comportamiento desinhibido tras sufrir un traumatismo craneoencefálico como consecuencia de un accidente de tráfico. En la TC craneal realizada de urgencia se objetiva una lesión quística frontal izquierda de gran tamaño con importante desplazamiento de línea media. Se punciona y evacua el contenido del quiste obteniéndose un líquido opalino rico en proteínas y elementos celulares no identificados. La RMN nos confirma los hallazgos radiológicos previos. El paciente es intervenido de forma reglada mediante craneotomía, evacuación completa del contenido y extirpación de las paredes de la lesión. El estudio anatomopatológico resulta ser compatible con el diagnóstico de quiste endodérmico.

Se han descrito casos de evolución agresiva con diseminación y recidiva tras manipulación quirúrgica de la lesión; por lo tanto, el tratamiento debe consistir en la extirpación completa de la misma. Para ello será necesario realizar el diagnóstico diferencial con otras lesiones quísticas intracraneales con el fin de adecuar el tratamiento a cada caso.

PALABRAS CLAVE: Quiste endodérmico. Quiste broncogénico. Sistema nervioso central.

Supratentorial endodermal cyst. Case report

Summary

Endodermal cysts (EC) of the central nervous system

Recibido: 5-02-07. Aceptado: 4-11-07 are very uncommon lesions predominantly located in the spinal canal. Although rare, intracranial EC have been mainly described in the posterior fossa, with the supratentorial location considered exceptional. Apart from the low frequency of these lesions, their pathoembriology still remais unknown. We report a patient with a huge frontal $\mathrm{EC}$ and review the literature.

A 62-year-old man presented with abnormal behaviour, disorientation and decreased level of consciousness after moderate head injury. Initial cranial CT scan revealed a large cyst in the left frontal region with marked midline shift. Emergency puncture and decompression of the cyst demonstrated a milky fluid with high protein levels. Cranial MRI after patient improvement confirmed the existence of the cystic lesion with less mass effect. Delayed surgery was performed with craniotomy and total removal of the cyst. Pathological examination confirmed the presence of a typical EC. Patient made a complete recovery on follow-up with no recurrence on postoperative MRIs.

Differential diagnosis of EC based on radiological data is quite difficult. As aggresive behaviour of this condition has been described following incomplete resections, the treatment of choice is a radical removal of the cyst in one or two stages depending on patient clinical condition.

KEY WORDS: Endodermal cyst. Bronchogenic cyst. Central nervous system.

\section{Introducción}

Los quistes endodérmicos intracraneales constituyen una entidad patológica muy poco frecuente que ha sido descrita preferentemente en la fosa posterior ${ }^{7,9,11-13,17,19,22-}$ 26,28 , siendo excepcionales los casos descritos a nivel supratentorial.

Gran parte del interés de estas lesiones radica en el hecho de que su embriología permanece aún desconocida ${ }^{4}$. A pesar de que son lesiones de naturaleza benigna, en ocasiones pueden presentar una diseminación extensa 


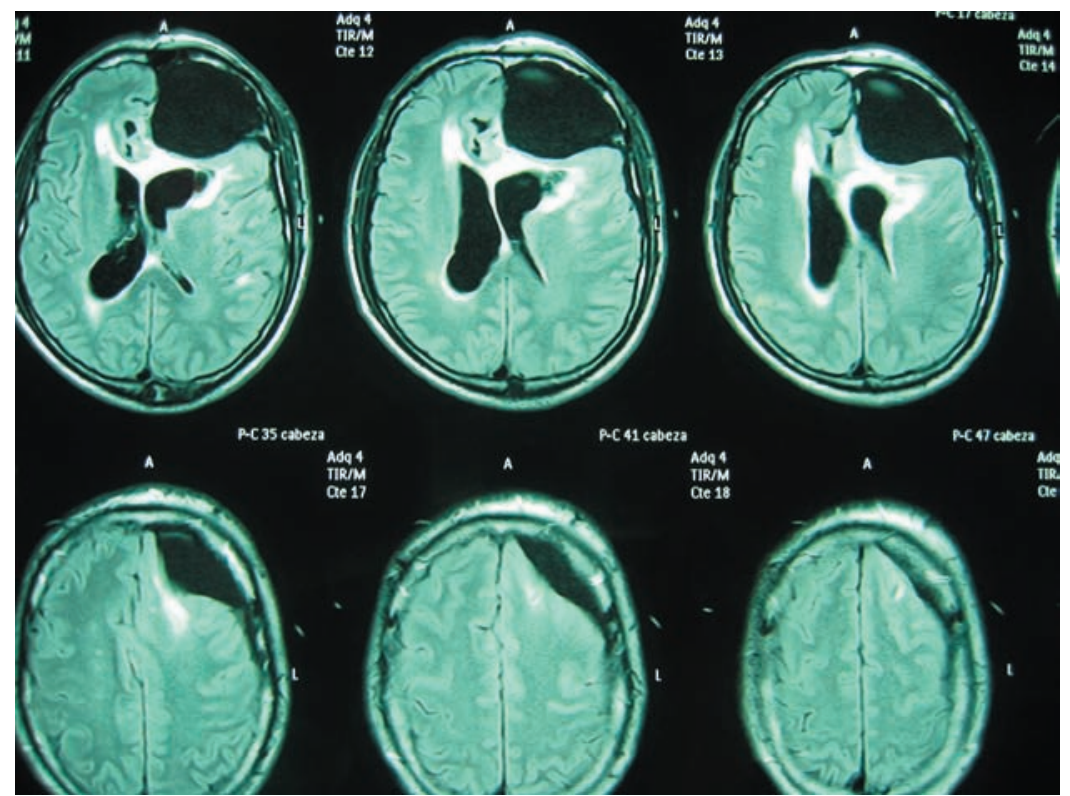

Figura 1. Cortes axiales de RMN craneal donde se aprecia una lesión quística extraparenquimatosa frontal izquierda de gran tamaño (a pesar de su punción y evacuación parcial previa). Las características radiológicas por sí solas no permiten asegurar el origen.

tras una resección incompleta con graves consecuencias clínicas $^{13,20,25}$. Debido a este riesgo de diseminación el tratamiento debe consistir en la extirpación completa de la lesión ${ }^{5}$. Para ello, es fundamental establecer el diagnóstico diferencial con otras lesiones quísticas a través de sus características morfológicas y en función de su localización específica en el encéfalo. Sin embargo, en muchos casos los hallazgos radiológicos no son específicos y el diagnóstico preoperatorio puede ser muy dificultoso. En concreto los quistes endodérmicos de tamaño considerable presentan marcadas similitudes radiológicas con los quistes aracnoideos gigantes ${ }^{4}$.

Se describe el caso de un paciente portador de un quiste endodérmico de grandes dimensiones y de localización supratentorial, y se plantea una revisión bibliográfica sobre este tipo de lesiones intracraneales.

\section{Caso clínico}

Se trata de un varón de 62 años que ingresa en el servicio de urgencias de nuestro hospital tras sufrir un accidente de tráfico con traumatismo craneoencefálico. El paciente no recuerda lo sucedido y se encuentra desorientado, agitado y agresivo. En la tomografía computarizada (TC) craneal realizada de urgencia se observa la existencia de una lesión hipodensa extraaxial frontal izquierda con importante efecto de masa, que condiciona una herniación subfacial y compresión de cisternas perimesencefálicas. Se le interviene de urgencia mediante punción de la lesión y colocación de un drenaje externo, tras lo cual el paciente experimenta una mejoría clínica significativa. El líquido extraído es opalino y su posterior análisis revela un alto contenido en proteínas $(2,5 \mathrm{~g} / \mathrm{L})$ y abundantes elementos celulares no identificados, descartándose sin embargo la existencia de microorganismos.

En la resonancia magnética $(\mathrm{RM})$ craneal practicada de forma programada se confirmaron los hallazgos de la TC (Figura 1).

El paciente es intervenido 10 días después mediante craneotomía para extirpación completa de la lesión. Durante la intervención se observa un quiste de paredes finas y transparentes, de localización extraaxial, que contiene un líquido xantocrómico. Se procede a la punción del mismo para extracción de su contenido líquido $\mathrm{y}$, posteriormente, se separan de forma meticulosa las paredes de la lesión de la duramadre y el parénquima cerebral subyacente (Figuras 2, 3 y 4).

El estudio anatomopatológico permite objetivar la existencia de un tejido de origen endodérmico constituido por un epitelio cúbico pseudoestratificado ciliado. Los hallazgos son compatibles con el diagnóstico de quiste broncogénico (Figura 5).

El paciente evoluciona favorablemente, mejorando su nivel de orientación así como la alteración de comportamiento. No se aprecian signos de focalidad neurológica en el postoperatorio. En la RMN de control realizada 6 meses después de la intervención se observa la reexpansión del tejido cerebral en ausencia de cavidad quística alguna (Figura 6).

\section{Discusión}

Los quistes endodérmicos representan una lesión tumoral muy poco frecuente. La mayoría de los que afectan al sistema nervioso central se sitúan a nivel espinal $^{1,18,23}$. Existen pocas series publicadas sobre quistes intracraneales, siendo los de localización supratentorial particularmente raros. La presentación de este caso clínico 

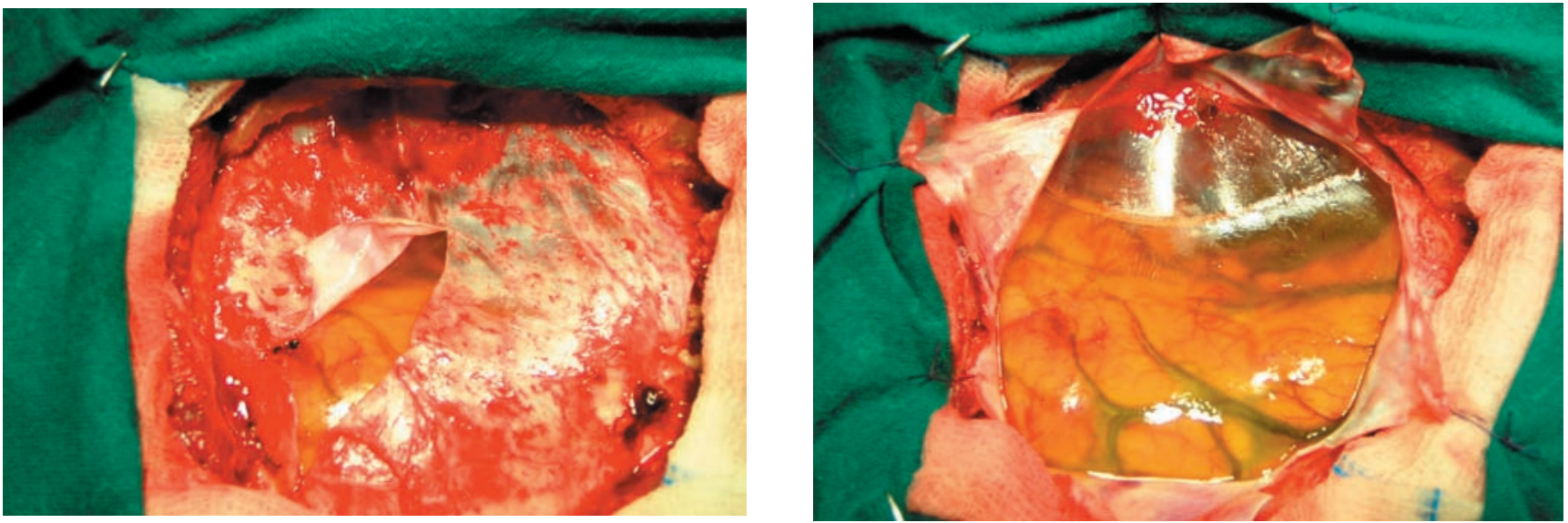

Figuras 2 y 3. Fotos quirúrgicas. Lesión de paredes finas y transparentes que contiene un líquido xantocrómico tras la evacuación previa del contenido opalino.

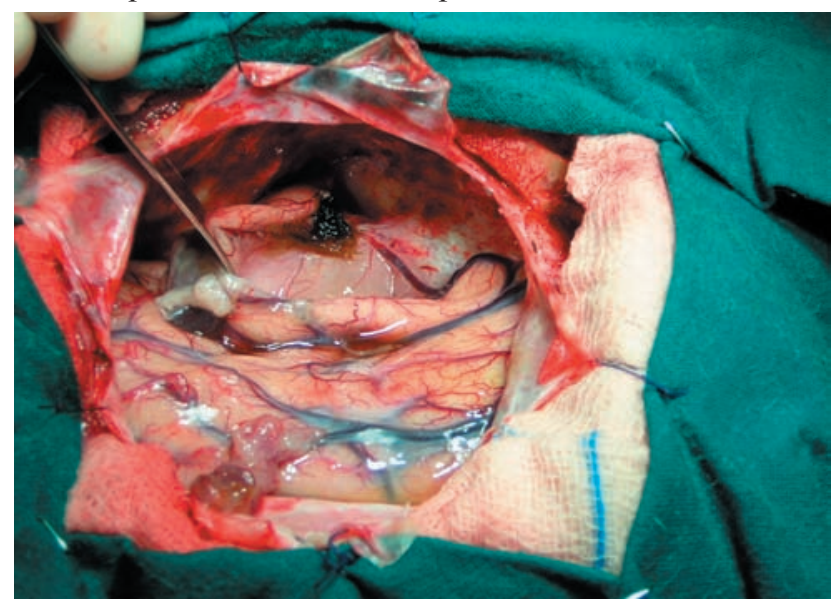

Figura 4. Cavidad resultante tras la extirpación de la lesión quistica frontal izquierda.

tiene por objeto ayudar en el proceso diagnóstico y manejo posterior de este tipo de lesiones.

Dado que los quistes endodérmicos intracraneales se han descrito en múltiples localizaciones, tales como la

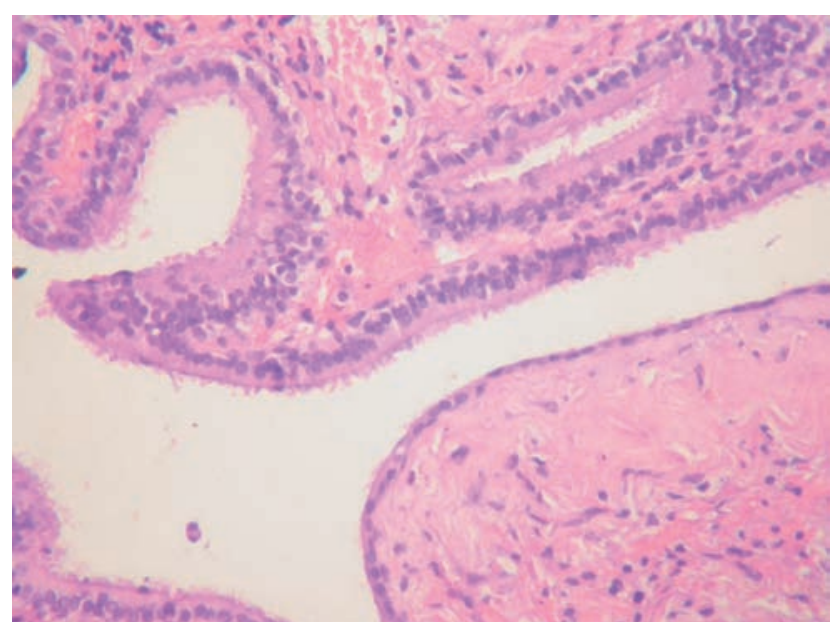

Figura 5. Corte histológico teñido con hematoxilina \& eosina que muestra las paredes de la lesión resecada. Se trata de un epitelio cúbico pseudoestratificado ciliado compatible con el diagnóstico de quiste broncogénico.

región bulbarr ${ }^{11,12}$, supraselar ${ }^{9}$ y convexidad cerebral ${ }^{4,9,16}$, septum pellucidum ${ }^{6} \mathrm{o}$ en la unión craneoespinal $^{8,27}$, exis-

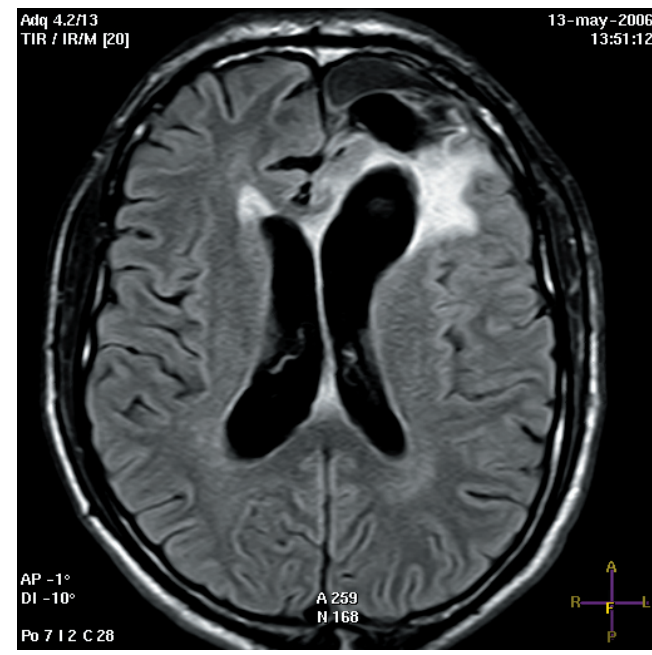

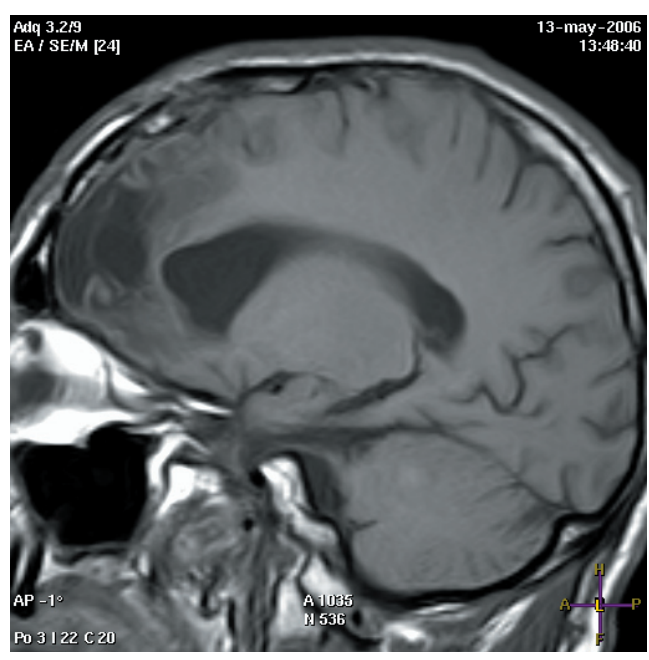

Figuras 6. RMN craneal de control realizada 6 meses tras la cirugía definitiva. Se objetiva la reexpansión del tejido cerebral subyacente. 
ten muy diversas presentaciones clínicas en función de las estructuras anatómicas próximas que puedan comprometer.

Se describe el caso de un varón portador de un quiste endodérmico supratentorial con una alteración del comportamiento mantenida en el tiempo, probablemente de años de evolución. El paciente experimenta un deterioro clínico de rápida evolución en relación con un traumatismo craneal secundario a un accidente de tráfico. Esta presentación clínica coincide con otros casos presentados en la literatura ${ }^{4,6}$, aunque en esta ocasión se añade como factor precipitante el traumatismo craneoencefálico. Se lleva a cabo un tratamiento en dos fases: la primera de ellas, debido al deterioro clínico brusco, consiste en la evacuación urgente de la lesión mediante su punción y colocación de un drenaje externo. El paciente experimenta una mejoría clínica significativa, recuperando un buen nivel de consciencia. Sin embargo, persiste un comportamiento desinhibido con una importante labilidad emocional. La segunda fase del tratamiento, la cual se realiza de modo programado 10 días después, consiste en la exéresis de las paredes de la lesión mediante la realización de una craneotomía frontal. Los hallazgos de la cirugía, coinciden con los descritos en la literatura en cuanto a las características morfológicas y contenido del quiste ${ }^{4}$. El paciente evoluciona favorablemente, remitiendo por completo su alteración del comportamiento. En la RMN craneal realizada 6 meses después de la cirugía se aprecia la ausencia de recidiva y diseminación, con una reexpansión casi completa del tejido cerebral perilesional.

La embriología de los quistes endodérmicos supratentoriales permanece aún desconocida. Es poco probable que su origen tenga lugar en remanentes endodérmicos de la notocorda puesto que el endodermo no se extiende más allá del mesencéfalo durante la embriogénesis ${ }^{4}$. Otros autores postulan que los quistes endodérmicos supratentoriales, al igual que otras lesiones quísticas supratentoriales tales como los quistes de la bolsa de Rathke y los quistes coloides, pueden provenir de restos de la bolsa de Seesel, un divertículo transitorio del intestino anterior durante el desarrollo embrionario.

Sin embargo, según Catala y Poirier $^{3}$, aunque el origen embriológico de los quistes coloides es probablemente endodérmico, se considera que aquellos derivados de la bolsa de Rathke están constituidos, al igual que ésta, de tejido ectodérmico.

Bavetta y cols ${ }^{2}$ establecen que aquellos casos localizados a nivel de la fosa craneal anterior pueden presentar características de diferenciación de la mucosa nasal y, por consiguiente, tendrían un origen ectodérmico.

Otros autores consideran la posibilidad de que su origen tenga lugar en una diferenciación alterada de células pluripotentes ectópicas.
Las lesiones quísticas intracraneales gigantes en los adultos no son frecuentes e incluyen diversas entidades patológicas: quistes aracnoideos, neuroepiteliales, coloides, dermoides, quistes parasitarios y tumores quísticos (ej. xantoastrocitomas pleomórficos, metástasis...). El diagnóstico deberá realizarse en base a criterios morfológicos macroscópicos y topográficos, mediante las pruebas de neuroimagen (TC y RMN), y a través del estudio histológico de las paredes de estas lesiones ${ }^{3,14,15,21,23}$. Preece y cols ${ }^{21}$ han presentado la gran variabilidad de hallazgos de TC y RM en 18 pacientes con quistes endodérmicos intracraneales, por lo que en muchos casos el diagnóstico no puede conocerse hasta identificar el epitelio característico en el estudio anatomopatológico de la lesión.

Se han descrito casos de diseminación y recidiva tras manipulación quirúrgica. Perry y cols ${ }^{20}$ describieron la diseminación agresiva craneoespinal de un quiste endodérmico tras punción y drenaje de la lesión. Existen otros casos de recidiva post-cirugía descritos en la literatura, aunque confinados a la fosa posterior, lugar de asiento de la lesión primaria ${ }^{13,25}$. Es sabido que la aspiración o escisión incompleta de quistes coloides y quistes derivados de la bolsa de Rathke también pueden dar lugar a recurrencias similares.

Por consiguiente, será fundamental realizar un diagnóstico diferencial con otras lesiones supratentoriales de naturaleza quística, con el fin de adecuar el tratamiento a cada caso $0^{4,5,13,20}$. En caso de tratarse de quistes endodérmicos será necesario realizar una extirpación completa para evitar la posibilidad de diseminación; teniendo en cuenta que puede ser necesario realizar un tratamiento quirúrgico en dos fases, en función de la presentación clínica.

\section{Conclusiones}

Los quistes endodérmicos supratentoriales constituyen lesiones muy poco frecuentes. Su origen sigue siendo desconocido. Teniendo en cuenta su potencial de diseminación, el tratamiento quirúrgico definitivo debe consistir en la extirpación completa de la lesión y, por consiguiente, será necesario realizar un diagnóstico diferencial con otras lesiones quísticas para instaurar el tratamiento oportuno.

Considerando que, en determinadas ocasiones, estas lesiones pueden alcanzar grandes dimensiones y comprometer la vida del paciente, el tratamiento podrá plantearse en dos fases: una primera fase de descompresión urgente, mediante punción de la lesión, seguida de un tratamiento diferido mediante craneotomía para la resección completa de las paredes del quiste. Este abordaje se ha mostrado exitoso en el caso presentado. 


\section{Bibliografía}

1. Agrillo, A., Passacantilli, E., Santero, A., Delfín, R.: Spinal intradural endodermal cyst located anterior to the cervical spinal cord. J Neuroradiol. 1994; 21: 262-266.

2. Bavetta, S., El-Shunnar, K., Hamlyn, P.J.: Neurenteric cyst of the anterior cranial fossa. Br J Neurosurg. 1996; 10: 225-227.

3. Catala, M., Poirier, J.: Intracranial and intraspinal epithelial cysts: A simplified classification. Rev Neurol (Paris). 2000; 156: 447-449.

4. Christov, C., Chretien, F., Brugieres, P., Djindjian, M.: Giant supratentorial enterogenous cyst: report of a case, literature review, and discussion of pathogenesis. Neurosurgery. 2004; 54: 759-763.

5. Du, Y.D., Cao, L.N., Zhao, G.: The treatment of enterogenous cyst: a report of 12 cases. Zhonghua Wai Ke Za Zhi. 1994; 32: 49-50.

6. Hamlat, A., Casallo-Quiliano, C., Saikali, S., Adn, M., Brassier, G.: Huge colloid cyst: case report and review of inusual forms. Neurosurgery. 1999; 44: 401-404.

7. Ito, S., Fujiwara, S., Mizoi, K., Namiki, T., Yosimoto, T.: Enterogenous cyst at the cerebellopontine angle: case report. Surg Neurol. 1992; 37: 366-370.

8. Kak, V.K., Gupta, R.K., Sharma, B.S., Banerjee, A.K.: Craniospinal enterogenous cyst: case report. J Neurosurg Sci. 2001; 45: 220-223.

9. Keyaki, A., Hirano, A., Llena ,J.F.: Differential diagnosis and origin of epithelial cysts in the central nervous system: report of seven cases and review of literature. Acta Neurochir (Wien). 2004; 146: 397-401.

11. Lach, B., Russell, N., Atack, D., Benoit, B.: Intraparenchymal epithelial (enterogenous) cyst of the medulla oblongata. Can J Neurol Sci. 1989; 16: 206-210.

12. Lin, J., Feng, H., Li, F., Chen, Z., Wu, G.: Ventral brainstem enterogenous cyst: an unusual location. Acta Neurochir (Wien). 2004; 146: 419-420.

13. Malcolm, G.P., Symon, L., Kendall, B., Pires, M.: Intracranial neurenteric cysts: Report of two cases. J Neurosurg. 1991; 75: 115-120.

14. Miyagami, M., Kasahara, E., Miyazaki, S., Tsubokawa, T., Kagawa, Y.: Ultrastructural findings of arachnoid cysts and epithelial cysts. No To Shinkei. 1989; 41: 411-418.

15. Morimura, T., Maeda, Y., Tani, E., Nishigami, T.: Immunohistochemical differential diagnosis of benign cysts in the central nervous system. Noshuyo Byori. 1994; 11: 7-13.
16. Motohashi, O., Kameyama, M., Imaizumi, S., Mino, M., Naganuma, H., Onuma, T.: An epithelial cyst of the frontal convexity. No To Shinkei. 1991; 43: 545-553.

17. Nakagawa, Y., Suzuki, K., Tatebe, A., Andachi, H.: Epithelial cyst in the cerebellopontine angle. Neurol Med Chir (Tokyo). 1996; 36: 251-254.

18. Nalm-ur-Rahman, Jamjoom, A., al-Rajeh, S.M., alSohaibani, M.O.: Spinal intradural extramedullary enterogenous cysts. Report of two cases and review of literature. J Clin Neurosci. 2001; 8: 133-139.

19. Okabe, S., Kamata, K., Kohno, T., Harada, Y.: Enterogenous cyst in the fourth ventricle: case report. Neurol Med Chir (Tokyo). 1995; 35: 40-44.

20. Perry, A., Scheithauer, B.W., Zaias, B.W., Minassian, H.V.; Aggressive enterogenous cyst with extensive craniospinal spread: case report. Neurosurgery. 1999; 44: 401-404.

21. Preece, M.T., Osborn, A.G., Chin, S.S., Smirniotopoulos, J.G.: Intracranial neuroenteric cysts: imaging and pathology spectrum. AJNR. 2006; 27: 1211-1216.

22. Ray, A., Chakraborty, A., Donaldson-Hugh, M.: Enterogenous cyst of the posterior fossa. Br J Neurosurg. 2000; 14: 249-251.

23. Shuangshoti, S., Netsky, M.G.: Neuroepithelial (colloid) cysts of the nervous system: Further observations on pathogenesis, location, incidence, and histochemistry. Neurology. 1966; 16: 887-903.

24. Umezu, H., Aiba, T., Unakami, M.: Enterogenous cyst of the cerebellopontine angle cistern: case report. Neurosurgery. 1991; 28: 462-465.

25. Vaishya, S., Ramesh, T.: Spontaneous relapsing and recurring large multiloculated posterior fossa enteric cyst. Acta Neurochir (Wien). 2006; 148: 985-988.

26. Van der Wal, A.C., Troost, D.: Enterogenous cyst of the brainstem: a case report. Neuropediatrics. 1988; 19: 216217.

27. Wagner, H.J., Seidel, A., Reusche, E., Sepehrnia, A., Kruse, K., Sperner, J.: A craniospinal enterogenous cyst: case report. Neuropediatrics. 1998; 29: 212-214.

28. Wang, Y.C., Chiang, Y.H., Chiou, S.Y., Fu, Y.M., Lee, W.W.: Enterogenous cyst of the fourth ventricle: case report. Zhonghua Yi Xue Za Zhi (Taipei). 1992; 50: 331-334.

Garbizu, J.M.; Mateo-Sierra, O.; Iza, B.; Ruiz-Juretschke, F.; Pérez-Calvo, J.M.: Quiste endodérmico supratentorial: caso clínico y revisión de la literatura. Neurocirugía 2009; 20: $367-371$. 